\title{
Journal of the American Society of Echocardiography
}

Copy of e-mail Notification

RUSH: Journal of the American Society of Echocardiography article for proofing (\# 778)

$====$

Dear Author,

The proof of your article, to be published by Elsevier in the Journal of the American Society of Echocardiography, is available as a "PDF" file at the following URL:

http://rapidproof.cadmus.com/RapidProof/retrieval/index.jsp

Login: your e-mail address

Password: ----

The site contains 1 file. You will need to have Adobe Acrobat Reader software to read this file. This is free software and is available for user download at: http://www.adobe.com/products/acrobat/readstep.html

After accessing the PDF file, please:

1) Carefully proofread the entire article, including any tables, equations, figure legends and references.

2) Ensure that your affiliations and address are correct and complete.

3) Check that any Greek letter, especially "mu," has translated correctly.

4) Verify all scientific notations, drug dosages, and names and locations of manufacturers.

5) Be sure permission has been procured for any reprinted material.

6)Answer all author queries completely. They are listed on the last page of the proof.

You may choose to list the corrections (including the replies to any queries) in an e-mail and return to me using the "reply" button. Using this option, please refer to the line numbers on the proof. If, for any reason, this is not possible, mark the corrections and any other comments (including replies to questions) on a printout of the PDF file and fax this to Efraim Salzberg (fax \#: 215-239-3388), or mail to the address given below.

Do not attempt to edit the PDF file (including adding Post-it type notes).

Within 48 hours, please return the following to the address given below:

1) Corrected PDF set of page proofs

2) Print quality hard copy figures for corrections if necessary (we CANNOT accept figures on disk at this stage). If your article contains color illustrations and you would like to receive proofs of these illustrations, please contact us within 48 hours.

If you submitted usable color figures with your article they will appear in color on the web, at no extra charge, as you can see in the attached PDF proof of your article. In the printed issue, color reproduction depends on journal policy and whether or not you agree to bear any costs.

If you have any problems or questions, please contact me. PLEASE ALWAYS INCLUDE YOUR ARTICLE NUMBER (778) WITH ALL CORRESPONDENCE.

Sincerely,

Efraim Salzberg

Issue Manager

Elsevier Science

1600 JFK Blvd, Suite 1800

Philadelphia, PA 19103-2899 USA

Phone: 215-239-3408

Fax: 215-239-3388 


\section{E.Salzberg@elsevier.com}



Left Ventricular Volume Measurements by Contrast-enhanced Real-time 3-Dimensional Echocardiography

\author{
Enrico G. Caiani, PhD, Patrick Coon, BS, RDCS, Cristiana Corsi, PhD, Sascha Goonewardena, MD, \\ Dianna Bardo, MD, Patrick Rafter, MS, Lissa Sugeng, MD, Victor Mor-Avi, PhD, and \\ Roberto M. Lang, MD, Chicago, Illinois; Milan and Bologna, Italy; \\ and Andover, Massachusetts
}

\begin{abstract}
Real-time 3-dimensional echocardiographic continuous imaging (CI) with contrast underestimates left ventricular (LV) volumes. We studied the effects of dual-triggered (DT) acquisition on the accuracy of LV volume measurements for patients with poor acoustic windows. Real-time 3-dimensional echocardiographic imaging was performed in 20 patients during LV opacification (Definity) on the same day as cardiac magnetic resonance imaging. Both CI and DT data were analyzed using custom software to calculate end-systolic volume (ESV) and end-diastolic volume (EDV), which were compared with the cardiac mag-
\end{abstract}

Accurate quantification of left ventricular (LV) volumes and ejection fraction (EF) is essential in clinical decision making and follow-up assessment. ${ }^{1-3}$ Today, the most commonly used imaging modality to assess LV function is 2-dimensional (2D) echocardiography (2DE). ${ }^{4}$ Nevertheless, 2DE estimates of $L V$ volumes and $\mathrm{EF}$ rely on the ability to visualize the entire LV endocardium in nonforeshortened apical views and are based on geometric assumptions, which can introduce errors, especially in odd-shaped ventricles or in the presence of wall-motion abnormalities. ${ }^{5-8}$ The recently developed transthoracic real-time 3-dimensional (3D) echocardiographic (RT3DE) imaging with full matrix-array technology has the potential to overcome these limitations. Although LV volume

From the Noninvasive Cardiac Imaging Laboratory, University of Chicago (E.G.C., P.C., S.G., D.B., L.S., V.M-A., R.M.L.); Dipartimento di Bioingegneria, Politecnico di Milano (E.G.C.); DEIS, Università di Bologna (C.C.); and Philips Medical Systems, Andover, Massachusetts (P.R.).

Supported by the American Society of Echocardiography Sonographer's Grant (P.C.) and a research grant from Bristol-Myers Squibb.

Reprint requests: Roberto M. Lang, MD, University of Chicago Medical Center, MC5084, 5841 S Maryland Ave, Chicago, IL 60637 (E-mail: rlang@medicine.bsd.uchicago.edu).

$0894-7317 / \$ 30.00$

Copyright 2005 by the American Society of Echocardiography. doi:10.1016/j.echo.2005.06.008 netic resonance reference. CI correlated well with the cardiac magnetic resonance reference (EDV: $r=0.89$; ESV: $r=0.93$ ), but underestimated EDV and ESV by $17 \%$ and $19 \%$, respectively. In contrast, DT resulted in higher correlation (EDV: $r=0.95$; ESV: $r=0.96$ ) and smaller biases ( $9 \%$ and $6 \%$, respectively). In conclusion, because the accuracy of $\mathrm{LV}$ volume measurements depends on the acquisition strategy of contrast-enhanced real-time 3-dimensional echocardiographic images, the use of DT instead of the conventional $\mathrm{CI}$ acquisition is recommended. ( $\mathrm{J}$ Am Soc Echocardiogr 2005;xx:xxx.)

and EF measurements obtained from RT3DE data sets are more accurate than those derived from $2 \mathrm{DE},{ }^{9-11}$ their accuracy depends on image quality and the ability to visualize the LV endocardium in multiple cut planes, which are limited in approximately $20 \%$ to $30 \%$ of consecutive patients. ${ }^{12}$

LV opacification is routinely used with $2 \mathrm{DE}$ to improve endocardial visualization and, thus, increase the accuracy of LV function assessment for patients with poor acoustic windows. ${ }^{13-18}$ To date, the feasibility of LV volume quantification from contrastenhanced RT3DE data sets in these patients has not been tested, and the potential improvement in the accuracy of LV volume measurements remains hypothetical. In fact, in our recent study, ${ }^{11}$ we found that contrast enhancement in patients with good image quality unexpectedly reduced the accuracy of RT3DE-derived LV volume measurements, which were consistently underestimated compared with both nonenhanced RT3DE and cardiac magnetic resonance (CMR) measurements, suggesting increased bubble destruction by the high density of scan lines and increased duration of bubble exposure with volumetric imaging.

In this study, we hypothesized that contrastenhanced RT3DE image acquisition using selective end-diastolic (ED) and end-systolic (ES) dual triggering (DT) instead of continuous imaging (CI) would reduce bubble destruction and, thus, improve the 
accuracy of LV volume measurements. Accordingly, our aim was to evaluate the effects of this acquisition strategy on the accuracy of LV volume and $\mathrm{EF}$ measurements against a CMR reference for patients with poor acoustic windows who require contrast enhancement for endocardial visualization.

\section{METHODS}

\section{Study Design}

Patients referred for CMR evaluation of LV function were studied.

Exclusion criteria were: dyspnea precluding a 10- to 15-second breath hold, cardiac arrhythmias, left bundle branch block, prior sternotomy, pacemaker or defibrillator implantation, claustrophobia, and other known contraindications to CMR imaging. Before enrollment, patients were screened by $2 \mathrm{D}$ transthoracic echocardiography. Patients with suboptimal acoustic windows, defined as inadequate LV endocardial visualization in at least two contiguous segments in each of the 3 conventional 2D apical views, were included in the study. Using these criteria, 20 patients were enrolled (age $58 \pm 17$ years; 10 men), including 7 patients with coronary artery disease, 9 with dilated cardiomyopathy, 2 with hypertrophic cardiomyopathy, 1 with pulmonary hypertension, and 1 with normal LV function. Written informed consent was obtained from all patients. The RT3DE examination was performed on the same day as the CMR study.

\section{CMR Imaging}

Images were acquired with a 1.5-T scanner (General Electric, Milwaukee, Wis) with a phased-array cardiac coil. Electrocardiographically gated localizing spin-echo sequences were used to identify the long axis of the heart to allow imaging in anatomically correct short-axis slices. Steady-state free precession dynamic gradient-echo cineloops were obtained during a 10- to 15 -second breath hold with a temporal resolution of 20 frames per cardiac cycle. In all patients, 6 to 10 cineloops were obtained at different LV levels from the atrioventricular ring to the LV apex (9-mm slice thickness, no gaps).

\section{RT3DE Imaging}

Contrast-enhanced RT3DE imaging was performed using a commercial ultrasound scanner (Sonos 7500, Philips Medical Systems, Andover, Mass) equipped with a full matrixarray transducer (X4, 2-4 MHz) from the apical windows during intravenous infusion of the contrast agent Definity (1.3 $\mathrm{mL}$ in $50 \mathrm{~mL}$ normal saline, at $4.0 \mathrm{~mL} / \mathrm{min}$ ). Imaging was performed in the harmonic mode at low mechanical index (0.3-0.4). The 3D images were optimized and special care was taken to include the entire LV cavity within the pyramidal scan volume. Wide-angled acquisition of the entire LV was performed by separately acquiring 4 subvolumes over 8 consecutive cardiac cycles. First, RT3DE data set was acquired during $\mathrm{CI}$. Then, two triggers were set to the electrocardiographic $R$ wave and the end of the $T$ wave for ED and ES acquisitions, respectively, and a DT data set including only ED and ES frames was obtained. For both strategies, acquisition was performed during a breath hold to minimize motion artifacts. In addition, at the end of the protocol, a nonenhanced RT3DE data set was acquired in CI mode.

\section{CMR Image Analysis}

Images were analyzed using commercial software (MASS Analysis, General Electric). Initially, LV slices were selected for analysis beginning with the highest basal slice where the LV outflow tract was not visible, and ending with the lowest apical slice where the LV cavity was visualized. Endocardial contours were traced semiautomatically in every frame of each slice including the papillary muscles in the LV cavity. From these contours, LV volume was computed throughout the cardiac cycle using a disk-area summation method (modified Simpson's rule). The ED volume (EDV) and ES volume (ESV) were determined as the maximum and the minimum volumes reached during the cardiac cycle, respectively, and used to compute the EF, as $100 \times(\mathrm{EDV}-\mathrm{ESV}) / \mathrm{EDV}$. The CMR data served as the gold standard for comparisons against contrast-enhanced RT3DE data. All tracings were performed by an investigator experienced in CMR analysis who had no knowledge of the echocardiographic measurements.

\section{Contrast-enhanced RT3DE Volume Calculations}

The contrast-enhanced RT3DE data sets were analyzed using custom software ${ }^{11}$ for semiautomated LV surface detection based on the level-set approach. ${ }^{19-21}$ First, the ED and ES frames, visually determined as the largest and smallest LV cavities in the cardiac cycle, were selected for analysis. LV long axis was manually identified and LV surface was initialized in 4 evenly rotated long-axis imaging planes (45-degree step) by manually selecting two points at each of 6 to 8 different depths from apex to base (Figure 1). To be consistent with the CMR tracings, papillary muscles when visible were included in the LV cavity. Subsequently, the verification and adjustment of the selected points was performed in the short-axis planes, at each of the previously initialized depths. The final LV endocardial surface (Figure 1) was obtained as the solution of the partial differential equation guiding the level-set function, using the manually initialized surface as the initial condition. The LV volume was measured from the number of voxels inside the detected surface, and EF calculated using the same formula as used for the CMR measurements.

\section{RT3DE LV Long-axis Dimension Measurement}

To evaluate the effects of contrast enhancement and different acquisition strategies on the LV long-axis dimension, ED and ES frames from both nonenhanced RT3DE, and CI and DT contrast-enhanced data sets, were analyzed 

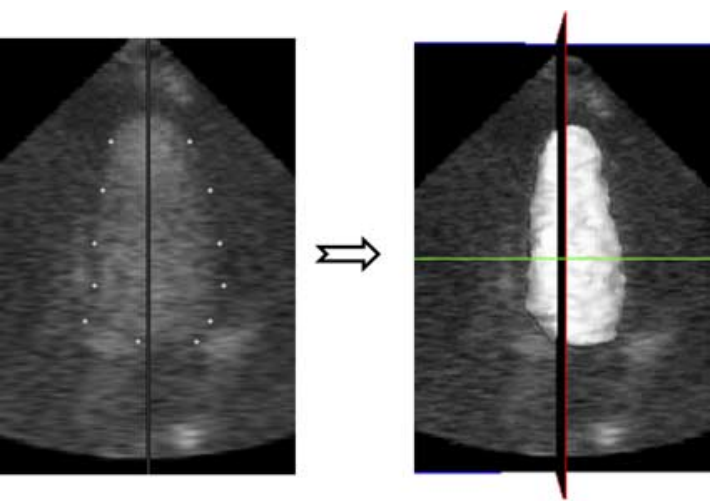

Figure 1 Left, Cut plane of left ventricle (LV) extracted from contrast-enhanced real-time 3-dimensional echocardiographic end-systolic data set obtained with dual triggering shown with set of points defined during manual initialization of endocardial surface. Dark gray vertical line, Long axis of ventricle. Right, LV endocardial surface obtained from initialized points using level-set algorithm.

using commercial software (3DQ-QLab, Philips Medical Systems). Cut planes were manually manipulated to obtain the anatomically correct apical 2- and 4-chamber views of the LV with the largest long-axis dimension, as previously described. ${ }^{22}$ In each view, the points of insertion of the mitral leaflets into the annulus were connected by a straight line, and the LV long-axis dimension measured as the distance between the center of this line and the most distal apical endocardium. The LV long-axis dimension was then determined as the average of the apical 2- and 4-chamber measurements.

\section{Reproducibility}

To assess the reproducibility of EDV, ESV, and EF measurements from contrast-enhanced CI and DT RT3DE data sets, a subgroup of 10 patients was randomly selected. To determine the interobserver variability, data sets obtained in this subgroup of patients were analyzed by two independent observers blinded to the CMR measurements. The intraobserver variability was assessed by having the same data sets analyzed a second time after 1 month by one of the investigators, who was blinded to the results of the previous tracings and the CMR measurements.

\section{Statistical Analysis}

Values were expressed as mean $\pm \mathrm{SD}$. Contrast-enhanced CI and DT RT3DE measurements were compared with CMR values using paired $t$ test. Agreement between CI and DT values of EDV, ESV, and EF with the CMR reference values was evaluated using linear regression and Pearson's correlation coefficients. In addition, Bland-Altman analysis was performed to calculate the bias and limits of agreement, and paired $t$ tests were used to verify the significance of the biases. In addition, RT3DE EDV and ESV were combined to evaluate the relationship between the measurement errors and LV size. This was performed sepa-

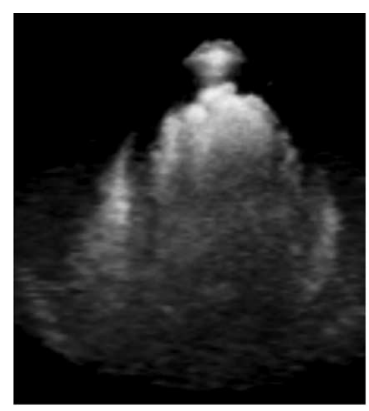

Continuous imaging
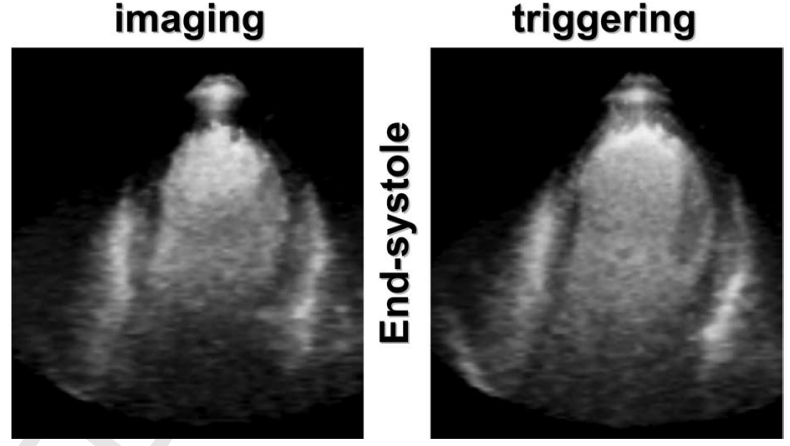

Figure 2 Slice extracted from two contrast-enhanced realtime 3-dimensional echocardiographic data sets acquired with continuous imaging (left) and selective dual triggering (right) in same patient at end diastole (top) and end systole (bottom). Images represent same anatomic slice.

rately for CI and DT by comparing LV volumes against the CMR reference using linear regression. Interobserver and intraobserver variability of the CI and DT measurements were calculated as the SD of the corresponding repeated measurements and expressed in percent of their mean. A 1-way analysis of variance for correlated samples and Tukey test were applied to verify the significance of the differences in the ED and ES LV long-axis measurements of the nonenhanced RT3DE and the contrast-enhanced CI and DT data.

\section{RESULTS}

CMR values for EDV and ESV ranged from 83 to 281 $\mathrm{mL}$ and from 26 to $188 \mathrm{~mL}$, respectively, whereas the $\mathrm{EF}$ ranged from $24 \%$ to $71 \%$. Acquisition of RT3DE data sets was feasible in all 20 patients enrolled in the study. The time required to analyze a single contrast-enhanced RT3DE data set, including data retrieval, frame selection, surface detection, and computation of LV volumes, was approximately 5 minutes.

Figure 2 shows an example of a slice extracted from two contrast-enhanced RT3DE data sets, acquired with $\mathrm{CI}$ and DT at ED and in the same patient. These images represent the same anatomic slice, with the only 
Table 1 The left ventricular end-diastolic, end-systolic long-axis dimension measured in 20 patients (mean \pm SD) from nonenhanced and contrast-enhanced real-time 3-dimensional echocardiography, with dual triggering and continuous imaging acquisition

\begin{tabular}{lll}
\hline & ED, $\mathbf{~ c m}$ & ES, cm \\
\hline RT3DE & $9.1 \pm 0.7^{*}$ & $8.2 \pm 1.1^{*}$ \\
Contrast-enhanced CI & $9.3 \pm 0.9^{*}$ & $8.2 \pm 1.0^{*}$ \\
Contrast-enhanced DT & $9.7 \pm 0.9$ & $8.6 \pm 1.0$
\end{tabular}

${ }^{*} P<.01,1$-way analysis of variance for correlated samples and Tukey test versus DT.

Table 2 Left ventricular end-diastolic and end-systolic volumes and ejection fraction measured in 20 patients (mean \pm SD) by cardiac magnetic resonance and contrast- enhanced real-time 3-dimensional echocardiography with dual triggering and with continuous imaging

\begin{tabular}{lllc}
\hline & EDV, $\mathbf{m L}$ & \multicolumn{1}{c}{ ESV, $\mathbf{m L}$} & EF, $\%$ \\
\hline CMR & $164 \pm 64$ & $94 \pm 55$ & $47 \pm 16$ \\
RT3DE DT & $150 \pm 65^{*}$ & $89 \pm 48$ & $42 \pm 17$ \\
RT3DE CI & $141 \pm 57^{*}$ & $79 \pm 48^{*}$ & $47 \pm 16$
\end{tabular}

${ }^{*} P<.05$, paired $t$ test compared with CMR values.

difference being the acquisition strategy. Bubble destruction, reflected by the apparent smaller size of the opacified LV cavity, is more pronounced in the CI images compared with the DT images. In addition, this increased destruction is more pronounced at ED than at ES for both acquisition modes. As a result, the mean LV long-axis dimensions measured in all patients were smaller with CI than DT (Table 1). Of note, compared with contrast-enhanced DT, ED and ES LV long-axis dimensions were significantly underestimated for both contrast-enhanced CI (ED frame: $-0.41 \mathrm{~cm}, P<.01$; ES frame: $-0.39 \mathrm{~cm}, P=.02)$ and nonenhanced RT3DE (ED frame: $-0.68 \mathrm{~cm}, P<.01$; ES frame: $-0.41 \mathrm{~cm}, P<.01)$.

The mean values of $\mathrm{LV}$ volumes and $\mathrm{EF}$ obtained with each technique (ie, CI, DT, and CMR) are presented in Table 2. A significant underestimation in both EDV and ESV compared with CMR was noted for CI, whereas DT was found to underestimate only the EDV. No significant differences were found in EF values. DT-derived measurements showed better correlation with CMR (EDV: $r=0.95$, standard error of the estimate $[\mathrm{SEE}]=21 \mathrm{~mL}$; ESV: $r=0.96$, SEE $=14$ $\mathrm{mL}$; $\mathrm{EF}: r=0.77, \mathrm{SEE}=11 \%$ ) compared with the CI values (EDV: $r=0.89$, SEE $=27 \mathrm{~mL}$; ESV: $r=0.93$, SEE $=18 \mathrm{~mL}$; EF: $r=0.77$, SEE $=10 \%$ ) (Figures 3 to 5 ).

With CI, Bland-Altman analysis showed a significant underestimation $(P<.01)$ compared with CMR values in both $\mathrm{EDV}$, with a bias of $-23 \mathrm{~mL}(17 \%$ of the mean) and $95 \%$ limits of agreement at $\pm 59 \mathrm{~mL}$, and ESV, with a bias of $-15 \mathrm{~mL}$ (19\% of the mean) and $95 \%$ limits of agreement at $\pm 41 \mathrm{~mL}$. Conversely,
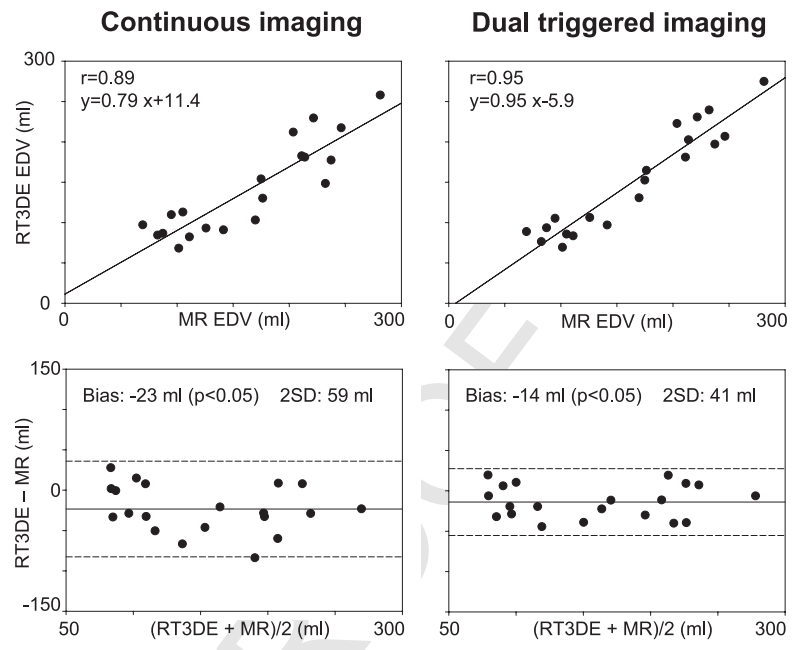

Figure 3 Linear regression (top) and Bland-Altman analysis (bottom) of left ventricular end-diastolic volume (EDV) measured in 20 patients from contrast-enhanced real-time 3-dimensional echocardiographic (RT3DE) data sets acquired with continuous imaging (left) and selective dual triggering (right) against cardiac magnetic resonance reference. Horizontal dashed lines, 95\% limits of agreement.

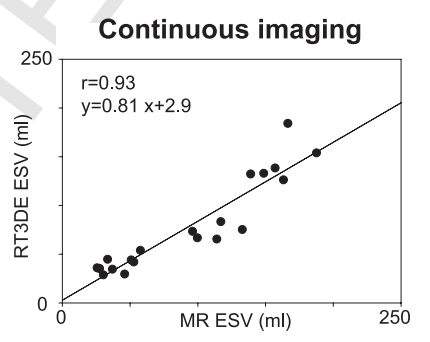

\section{Dual triggered imaging}
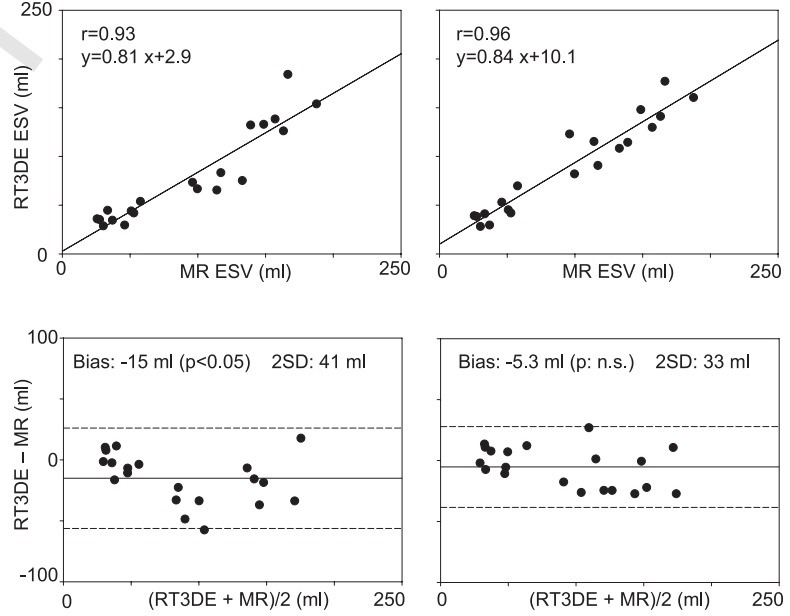

Figure 4 Linear regression (top) and Bland-Altman analysis (bottom) of left ventricular end-systolic volume (ESV) measured in 20 patients from contrast-enhanced real-time 3-dimensional echocardiographic (RT3DE) data sets acquired with continuous imaging (left) and selective dual triggering (right), against cardiac magnetic resonance reference. Horizontal dashed lines, 95\% limits of agreement.

EF showed zero bias with 95\% limits of agreement of $\pm 22 \%$ (Figures 3 to 5 ). DT values resulted in smaller biases compared with CMR in LV volumes (EDV: $-14 \mathrm{~mL}$, equal to $9 \%$ of the mean; ESV: $-5.3 \mathrm{~mL}$, equal to $6 \%$ of the mean), with a significant underestimation $(P<.01)$ in EDV only. In addition, the 95\% limits of agreement were narrower (EDV: \pm 41 

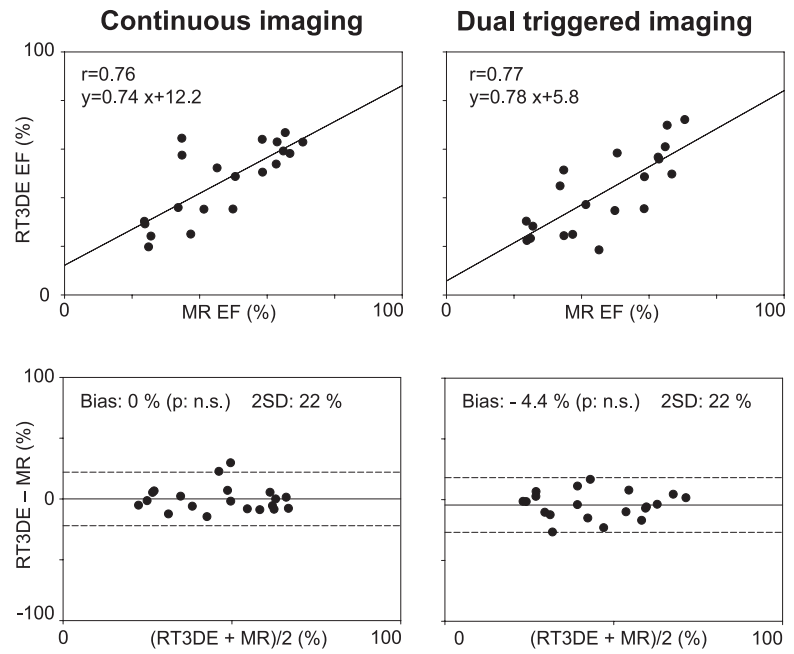

Figure 5 Linear regression (top) and Bland-Altman analysis (bottom) of left ventricular ejection fraction $(E F)$ measured in 20 patients from contrast-enhanced real-time 3-dimensional echocardiographic $(R T 3 D E)$ data sets acquired with continuous imaging (left) and selective dual triggering (right), against cardiac magnetic resonance reference. Horizontal dashed lines, 95\% limits of agreement.

$\mathrm{mL}, \mathrm{ESV}: \pm 33 \mathrm{~mL}$ ) than those of CI measurements. With DT, EF showed a nonsignificant bias of $-4.4 \%$ with $95 \%$ limits of agreement of $22 \%$.

Figure 6 shows the linear regression of the combined EDV and ESV measurement errors plotted against the CMR values, separately for CI and DT. Interestingly, CI-related errors showed a trend toward increasing underestimation with increasing LV size $(r=0.48$, SEE $=22.6)$. This observation was less pronounced with DT data $(r=0.36$, SEE $=$ 17.9).

The interobserver variability in EDV, ESV, and EF was similar for both acquisition strategies (CI: 8.2\%, $11.8 \%, 10.2 \%$, respectively; DT: $7.7 \%, 12.4 \%, 12.6 \%$, respectively). The intraobserver variability in EDV, ESV, and EF also showed similar values for both CI (6.1\%, 10.8\%, 10.3\%, respectively) and DT (7.6\%, $7.9 \%, 11.4 \%$, respectively) measurements.

\section{DISCUSSION}

Currently, 2DE is the most commonly used imaging technique to estimate $\mathrm{LV}$ volumes and $\mathrm{EF}$, which is usually performed in a subjective ${ }^{23}$ and experiencedependent $^{24}$ manner. The recent development of a full matrix-array transducer technology, ${ }^{25}$ suitable for the acquisition of wide-angle pyramidal volume data sets with adequate spatial and time resolution, offers the opportunity to quantify LV dimensions and $\mathrm{EF}$ in a more accurate way compared with conventional $2 \mathrm{DE}^{26}$ However, commercially avail-
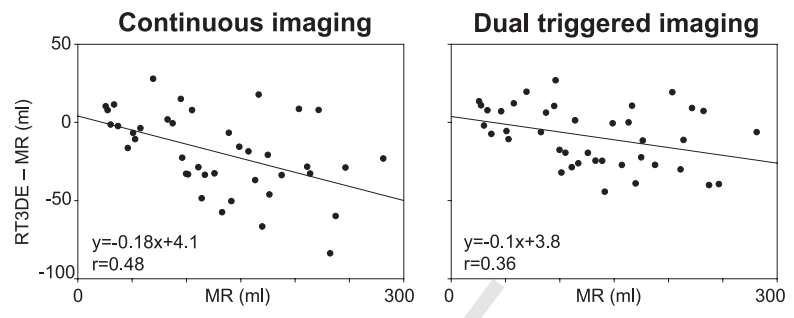

Figure 6 Linear regression between measurement errors in left ventricular volumes (combined end-systolic and end-diastolic data obtained in 20 patients) and cardiac magnetic resonance-derived volume values obtained from contrast-enhanced real-time 3-dimensional echocardiographic $(R T 3 D E)$ data sets: continuous imaging (left) and selective dual triggering (right).

able tools for analysis of RT3DE data sets still rely on manual or semiautomated tracing of LV endocardial borders on multiple $2 \mathrm{D}$ views extracted from the $3 \mathrm{D}$ data sets. ${ }^{9,10,27-29}$ Thus, the clinical applicability of this new technology still depends on the acoustic window of the patient, which determines the image quality of RT3DE data sets and the ability to visualize the endocardium in all cut planes.

LV opacification is a well-established technique for improving LV endocardial visualization, and, as a result, the accuracy of LV dimension measurements, which is widely used with 2DE imaging in patients with poor acoustic windows. ${ }^{13-18}$ An extension of this technique to RT3DE imaging could be expected to also improve the visualization of $\mathrm{LV}$ endocardial borders in these patients. However, to our knowledge, there are no data in the literature on improved accuracy in LV volume and EF measurements from RT3DE data sets with LV opacification. We previously observed that contrast-enhanced RT3DE data sets, obtained for patients with good acoustic windows, significantly underestimated LV volumes compared with nonenhanced RT3DE data sets, when using CMR data as a reference. We hypothesized that this observation was likely caused by increased bubble destruction with 3D scanning as a result of: (1) the high density of scan lines required to generate a 3D data set; and (2) the increased bubble exposure to ultrasound because each bubble traversing the heart is exposed as long as it is within the scanned volume rather than within a single plane.

The aim of this study was to test the accuracy of $\mathrm{LV}$ volumes and $\mathrm{EF}$ measurements obtained from contrast-enhanced RT3DE in a group of patients with poor acoustic windows, using two different image acquisition strategies: CI and selective DT. Our hypothesis was that DT acquisition of ED and ES frames would decrease bubble exposure to ultrasound and, thus, minimize bubble destruction and result in improved accuracy of LV volume measurements. 
To quantify LV volumes from contrast-enhanced RT3DE data sets, we used previously validated custom software ${ }^{11}$ capable of fast, semiautomated detection of LV endocardial surfaces, based on the level-set approach. ${ }^{19-21}$ Compared with other methodologies, ${ }^{9,22,30,31}$ this image segmentation technique is directly applied to the 3D data set, resulting in accurate detection of the 3D LV endocardial surface. This method was shown to provide excellent LV volume correlation with CMR values, together with reduced interobserver variability compared with manual tracings of $2 \mathrm{DE}$ images. ${ }^{11}$

We studied patients referred to CMR for assessment of LV function who had poor echocardiographic image quality precluding LV function evaluation without contrast enhancement. The poor quality of the nonenhanced RT3DE data sets was confirmed by visual inspection in multiple cut planes. Because of the inability to properly visualize the LV endocardium for the required manual initialization procedure, nonenhanced RT3DE data sets were not analyzed for $\mathrm{LV}$ volume quantification.

Both contrast-enhanced (CI and DT) and nonenhanced RT3DE data sets were analyzed to obtain the LV long-axis dimension. This was done to determine whether LV opacification could aid in visualizing the true LV apex and, thus, avoid foreshortened apical view from which LV length cannot be measured correctly. Our results showed that both nonenhanced and contrast-enhanced CI data resulted in underestimation of $\mathrm{LV}$ length in the $\mathrm{ED}$ and $\mathrm{ES}$ frames when compared with DT.

Moreover, our results showed that contrast-enhanced RT3DE consistently underestimated EDV, independently of the acquisition strategy, with the error being smaller with the DT approach compared with CI, probably as a result of cumulative effects of bubble destruction in consecutive frames. We demonstrated that the use of selective DT reduces bubble destruction at ES, resulting in a more accurate and nonbiased estimate of ESV compared with CI. Interestingly, EF was found to be not significantly different from CMR values in both acquisition strategies. Specifically, CI resulted in a zero bias, which could be explained by cancellation of simultaneous errors in EDV and ESV. Importantly, the reproducibility of the CI and DT volume measurements was similar, as reflected by interobserver and intraobserver variability of approximately $10 \%$, which is acceptable and common for clinically used techniques.

Linear regression of errors in combined LV EDV and ESV measurements compared with CMR showed a trend toward increased underestimation with increasing $L V$ volumes, indicating that bubble destruction was more pronounced farther away from the axis of the pyramid, which is probably related to the specific beam geometry. This trend, however, was less marked with DT compared with CI, providing addi- tional support to our hypothesis that this imaging strategy could allow more accurate measurements of $\mathrm{LV}$ volumes.

The use of CMR imaging as the standard reference technique could be viewed as a limitation of this study. Despite its superior endocardial definition, CMR imaging cannot provide perfectly accurate LV volume measurements, because these measurements are derived from discrete slices and rely on operator-dependent endocardial tracing. However, CMR is widely accepted as a standard reference technique for $\mathrm{LV}$ volume measurements. In addition, although RT3DE imaging is frequently referred to as a real-time technique, acquiring the entire $\mathrm{LV}$ in the wideangled mode requires separate acquisition of 4 subvolumes over 8 consecutive cardiac cycles. This may limit the applicability of this method for patients with severe dyspnea, atrial fibrillation, and cardiac arrhythmias. A methodologic limitation is that the semiautomated surface detection technique requires manual initialization, which may be viewed as providing the surface detection algorithm with some sort of LV shape information. Despite the fact that this is a subjective procedure, this technique was found to be reproducible and accurate. Nevertheless, further validation is necessary before attempted clinical use, including a more complete assessment of the reproducibility of RT3DE-derived measurements. Such assessment would require repeated data acquisitions in addition to repeated analyses, which was not performed in this study.

In summary, the accuracy of LV volume measurements from contrast-enhanced RT3DE data sets depends on image acquisition strategy. DT acquisition reduced bubbles destruction, thus, improving the visualization of the LV apex and increasing the accuracy of LV volume measurements. Therefore, the use of this acquisition strategy instead of the conventional $\mathrm{CI}$ is recommended.

\section{REFERENCES}

1. Cohen M, Packer M, Gorlin R. Indications for left-ventricular aneurysmectomy. Circulation 1983;67:717-22.

2. White HD, Norris RM, Brown MA, Brandt PWT, Whitlock RML, Wild CJ. Left-ventricular end-systolic volume as the major determinant of survival after recovery from myocardial infarction. Circulation 1987;76:44-51.

3. Wong M, Johnson G, Shabetai R, Hughes V, Bhat G, Lopez $\mathrm{B}$, et al. Echocardiographic variables as prognostic indicators and therapeutic monitors in chronic congestive heart failure: Veterans Affairs cooperative studies V-Heft-I and V-Heft-II. Circulation 1993;87:65-70

4. Schiller NB, Shah P, Crawford M, DeMaria A, Devereux R, Feigenbaum H, et al. Recommendations for quantitation of the left ventricle by two-dimensional echocardiography: American Society of Echocardiography committee on standards, subcommittee on quantitation of two-dimensional echocardiograms. J Am Soc Echocardiogr 1989;2:358-67. 
Journal of the American Society of Echocardiography

5. Teichholz LE, Kreulen T, Herman MV, Gorlin R. Problems in echocardiographic volume determinations: echocardiographicangiographic correlations in presence or absence of asynergy. Am J Cardiol 1976;37:7-11.

6. Folland ED, Parisi AF, Moynihan PF, Jones DR, Feldman CL, Tow DE. Assessment of left ventricular ejection fraction and volumes by real-time, 2-dimensional echocardiography: comparison of cineangiographic and radionuclide techniques. Circulation 1979;60:760-6.

7. Wyatt HL, Meerbaum S, Heng MK, Gueret P, Corday E. Cross-sectional echocardiography, 3 : analysis of mathematic models for quantifying volume of symmetric and asymmetric left ventricles. Am Heart J 1980;100:821-8.

8. Schnittger I, Fitzgerald PJ, Daughters GT, Ingels NB, Kantrowitz NE, Schwarzkopf A, et al. Limitations of comparing left ventricular volumes by 2 dimensional echocardiography, myocardial markers and cineangiography. Am J Cardiol 1982;50:512-9.

9. Kuhl HP, Schreckenberg M, Rulands D, Katoh M, Schafer W, Schummers G, et al. High-resolution transthoracic real-time three-dimensional echocardiography: quantitation of cardiac volumes and function using semi-automatic border detection and comparison with cardiac magnetic resonance imaging. J Am Coll Cardiol 2004;43:2083-90.

10. Jenkins C, Bricknell K, Hanekom L, Marwick TH. Reproducibility and accuracy of echocardiographic measurements of left ventricular parameters using real-time three-dimensional echocardiography. J Am Coll Cardiol 2004;44:878-86.

11. Caiani EG, Corsi C, Zamorano J, Sugeng L, MacEneaney P, Weinert $\mathrm{L}$, et al. Improved semi-automated quantification of left ventricular volumes and ejection fraction using threedimensional echocardiography with a full-matrix array transducer: comparison with MRI. J Am Soc Echocardiogr 2005

AQ: 5 (in press).
12. Yong Y, Wu D, Fernandes V, Kopelen HA, Shimoni S, Nagueh SF, et al. Diagnostic accuracy and cost-effectiveness of contrast echocardiography on evaluation of cardiac function in technically very difficult patients in the intensive care unit. Am J Cardiol 2002;89:711-8.

13. Firschke C, Koberl B, von Bibra H, Horcher J, Schomig A. Combined use of contrast-enhanced 2-dimensional and color Doppler echocardiography for improved left ventricular endocardial border delineation using Levovist, a new venous echocardiographic contrast agent. Int J Card Imaging 1997;13: 137-44.

14. Cohen JL, Cheirif J, Segar DS, Gillam LD, Gottdiener JS, Hausnerova E, et al. Improved left ventricular endocardial border delineation and opacification with OPTISON (FS069), a new echocardiographic contrast agent: results of a phase III multicenter trial. J Am Coll Cardiol 1998;32:746-52.

15. Zaglavara T, Norton M, Cumberledge B, Morris D, Irvine T, Cummins C, et al. Dobutamine stress echocardiography: improved endocardial border definition and wall motion analysis with tissue harmonic imaging. J Am Soc Echocardiogr 1999; 12:706-13.

16. Senior R, Andersson O, Caidahl K, Carlens P, Herregods MC, Jenni R, et al. Enhanced left ventricular endocardial border delineation with an intravenous injection of Sonovue, a new echocardiographic contrast agent: a European multicenter study. Echocardiography 2000;17:705-11.
17. Nagy A, Borbas S, Lengyel M. Measurement of left ventricular volumes and ejection fraction after intravenous contrast agent administration using standard echocardiographic equipment. Echocardiography 2000;17:433-7.

18. Dolan MS, Riad K, El Shafei A, Puri S, Tamirisa K, Bierig M, et al. Effect of intravenous contrast for left ventricular opacification and border definition on sensitivity and specificity of dobutamine stress echocardiography compared with coronary angiography in technically difficult patients. Am Heart J 2001; 142:908-15.

19. Osher S, Sethian JA. Fronts propagating with curvaturedependent speed: algorithms based on Hamilton-Jacobi formulations. J Comput Phys 1988;79:12-49.

20. Malladi R, Sethian JA, Vemuri BC. Shape modeling with front propagation: a level set approach. IEEE Trans Pattern Analysis Machine Intell 1995; 17:158-75.

21. Corsi C, Saracino G, Sarti A, Lamberti C. Left ventricular volume estimation for real-time three-dimensional echocardiography. IEEE Trans Med Imaging 2002;21:1202-8.

22. Mor-Avi V, Sugeng L, Weinert L, MacEneaney P, Caiani EG, Koch R, et al. Fast measurement of left ventricular mass with real-time three-dimensional echocardiography: comparison with magnetic resonance imaging. Circulation 2004;110:1814-8.

23. Popp R, Agatston A, Armstrong W, Nanda N, Pearlman A, Rakowski H, et al. Recommendations for training in performance and interpretation of stress echocardiography. J Am Soc Echocardiogr 1998;11:95-6.

24. Picano E, Lattanzi F, Orlandini A, Marini C, Labbate A. Stress echocardiography and the human factor: the importance of being expert. J Am Coll Cardiol 1991;17:666-9.

25. von Ramm OT. 2-D arrays. Ultrasound Med Biol 2000;26: S10-2.

26. Sugeng L, Weinert L, Lang RM. Left ventricular assessment using real time three dimensional echocardiography. Heart 2003;89:29-36.

27. Kuhl HP, Franke A, Janssens U, Merx M, Graf J, Krebs W, et al. Three-dimensional echocardiographic determination of left ventricular volumes and function by multiplane transesophageal transducer: dynamic in vitro validation and in vivo comparison with angiography and thermodilution. J Am Soc Echocardiogr 1998;11:1113-24.

28. Qin JJ, Jones M, Shiota T, Greenberg NL, Firstenberg MS, Tsujino H, et al. New digital measurement method for left ventricular volume using real-time three-dimensional echocardiography: comparison with electromagnetic flow method and magnetic resonance imaging. Eur J Echocardiogr 2000; 1:96-104.

29. Qin JX, Jones M, Shiota T, Greenberg NL, Tsujino H, Firstenberg MS, et al. Validation of real-time three-dimensional echocardiography for quantifying left ventricular volumes in the presence of a left ventricular aneurysm: in vitro and in vivo studies. J Am Coll Cardiol 2000;36:900-7.

30. Kuhl HP, Franke A, Merx M, Hoffmann R, Puschmann D, Hanrath P. Quantification of left ventricular function and mass using transesophageal three-dimensional echocardiography: validation of a method that uses long-axis cutplanes. Eur J Echocardiogr 2000;1:213-21.

31. Gerard O, Billon AC, Rouet JM, Jacob M, Fradkin M, Allouche C. Efficient model-based quantification of left ventricular function in 3-D echocardiography. IEEE Trans Med Imaging 2002;21:1059-68. 


\section{AUTHOR QUERIES}

\section{AUTHOR PLEASE ANSWER ALL QUERIES}

AQ6 - Please provide expansion for MR in legends for Figures 3 to 6.

AQ1- Please provide expansion for affiliation DEIS.

AQ2- CI is standard abbreviation for confidence interval. Please verify alternate use with Issue Manager or Editor.

AQ3 - Please expand all abbreviations used in Tables 1 and 2 within footnotes.

AQ4- Please verify expansion of SEE as standard error of the estimate.

AQ5- Please provide updated publication information for reference 11. 